\title{
Contribución de preservantes naturales en la obtención de futuros alimentos, ligados a la alimentación saludable
}

\author{
Oscar Mauricio Juárez Orellana \\ Máster en Docencia Universitaria \\ Investigador, Universidad de Oriente, El Salvador \\ E-mail: omjuarez04@yahoo.es
}

Recepción: 23/02/2015

Aceptación: 8/03/2015

\section{Resumen}

La investigación se realizó mediante el asocio Universidad de Oriente, Universidad Católica de El Salvador y el Fondo de Investigación de Educación Superior (FIES). En ella se evaluó la utilización de preservantes naturales como orégano (Origanum vulgares), clavo de olor (Syzygium aromaticum), comino (Cuminum cyminum) y ajo (Allium sativum), como sustitutos totales o parciales en productos alimenticios elaborados a partir de transformados cárnicos de res (Bos taurus) y cerdo (Sus scrofa domestica), que se comercializan en presentaciones como salami, chorizo, jamón, jamonada, salchicha o mortadela.

Se experimentó con aceites esenciales, extraídos de hojas o semillas de las especies vegetales seleccionadas para la investigación: orégano (Origanum vulgares), clavo de olor (Syzygium aromaticum), comino (Cuminum cyminum), y ajo (Allium sativum). Las pruebas también incluyeron la utilización de hojas y semillas secas que se pulverizaron para ser incorporadas como aditivos sólidos.

La última etapa de esta investigación consistió en aplicar a los procesados cárnicos la nueva fórmula de persevantes natural, el Omega 3, 6 y 9 a partir de la semilla de chan (Salvia hispánica L.). Se busca que estos componentes de Omega ${ }^{1}$ brinden un aporte nutricional, que eliminen la necesidad de recurrir a aditivos químicos elaborados sintéticamente, ya que son de fuente natural, permitiendo establecer un alimento seguro y nutritivo.

\begin{abstract}
The research was conducted by Universidad de Oriente, Universidad Católica de El Salvador and Fondo de Investigación de Educación Superior (FIES). Use of natural preservatives such as oregano (Origanum vulgares), clove (Syzygium aromaticum), cumin (Cuminum cyminum) and garlic (Allium sativum) was evaluated, as total or partial substitutes in food products made from processed meat beef (Bos taurus) and pig (Sus scrofa domestica ), are commercialized in presentations such as salami, sausage, ham, jamonada, sausage or mortadella .
\end{abstract}

Also experimented with essential oils extracted from leaves or seeds of plant species selected for investigation: oregano (Origanum vulgares), clove (Syzygium aromaticum), cumin (Cuminum cyminum) and garlic (Allium sativum). The tests also included the use of leaves and dried seeds, which were pulverized to be incorporated as solid additives. The last stage of this research consisted in applying to processed meat the new formula of natural preservatives, Omega 1, 3, 6 and 9 from salvia seed (Salvia hispánica L.). It is intended to demonstrate that these Omega components provide a nutritional contribution, which eliminates the need for chemical synthetically produced additives, as they are a natural source, allowing establishing safe and nutritious food.

Key words: food, species, preservation, natural preservatives, meatpacking, nutrition

1. Componentes que se encuentran en semillas leguminosas, como el ajonjolí y el chan, que previenen enfermedades cardiovasculares 


\section{Introducción}

Los alimentos por su origen biológico presen$\tan$ una serie de transformaciones que no solo modifican sus características originales, sino que llegan a producir su deterioro. La conservación de alimentos busca alargar la vida útil del futuro alimenticio y mantener sus características organolépticas y nutricionales.

La carne y los procesados cárnicos son alimentos altamente perecederos, por lo que se necesita un máximo cuidado al prepararlos y ser conservados, a fin de evitar que sean atacados por microorganismos que originen su descomposición. Para alargar la vida de los procesados cárnicos de res y cerdo, que se consumen popularmente en pizzas, emparedados, perros calientes, frituras, asados al carbón, al vapor o crudos, etc., es necesario conservarlos a bajas temperaturas en los anaqueles durante un periodo estimado de un mes.

Es de tener en cuenta que cuando se muele la carne aumenta la superficie de contacto, siendo más sensible a las posibles contaminaciones microbianas por el entorno, la manipulación, los utensilios, etc. Los tipos y la cantidad de microorganismos dependen de las condiciones sanitarias del medio ambiente del cual proceden los procesados cárnicos, de la calidad microbiológica de algunos ingredientes, del cuidado de quien los procesa, la forma de manejar el producto, las condiciones posteriores a su almacenamiento y distribución del mismo. Entre los microorganismos que más frecuentemente se encuentran en estos productos están: Staphylococus áureas, Salmonella, Escherichia coli, Shigella spp, Yersinia enterolitica, Listeria monocytogenes.

La demanda actual de la sociedad por productos con menos aditivos químicos ha forzado a la industria de los procesados cárnicos a buscar formas de remover completamente el uso de persevantes químicos; o adoptar alternativas naturales para el mantenimiento y extensión de la vida útil de los alimentos. En el arte culinario se han utilizado plantas, hierbas y especias, que es el común apelativo del uso de hojas y semillas como el orégano (Origanum vulgares), clavo de olor (Syzygium aromaticum), comino (Cuminum cyminum) y ajo (Allium sativum).

Estos contienen un gran número de sustancias con propiedades que inhiben la actividad metabólica de bacterias, mohos y levaduras. La mayoría de estos compuestos con actividad microbiana son compuestos fenólicos, terpenos, alcoholes alifáticos, aldehídos, cetonas, ácidos e isoflavonoides

\section{Materiales y métodos}

Esta investigación se realizó en el laboratorio de Microbiología de la Facultad de Ciencias Agronómicas de la Universidad de Oriente, edificio Jaguar de Piedra, ubicado en el cantón El Obrajuelo, Municipio de Quelepa, San Miguel. En ella se estudió parcial o profunda- 
mente la capacidad inhibidora del crecimiento microbiano; buscando establecer una fórmula para los procesados cárnicos que incluyan una proporción de fibra, grasa, agua y condimentos, así como la incorporación de la concentración del persevante natural y del aditivo Omega 3, 6 y 9, producido a partir de una fuente natural. Se buscó, además, la admisión de este proceso en la normativa salvadoreña NSO 67.02.13:98 sobre Carne y Productos Cárnicos Embutidos Crudos y Cocidos. El objeto final de la investigación fue siempre la obtención de un alimento saludable y nutritivo.

Para realizar esta investigación se requirió de equipo, cristalería y materiales adecuados al proceso; además de la aplicación de técnicas gravimétricas, analíticas, cualitativas y cuantitativas, procesos de destilación simple, destilación por arrastre de vapor, separación por densidades y solubilidad; separación mediante extractor soxhlet, filtración al vacío y análisis microbiológico. Fue necesario emplear técnicas básicas de laboratorio y el respectivo protocolo de limpieza y sanitizacion del área de trabajo.

El método utilizado en esta investigación fue de tipo experimental: en este el investigador controla deliberadamente las variables para delimitar relaciones entre ellas, y se basa en la metodología científica. A través de él se recopilan datos para comparar las mediciones de comportamiento entre un grupo control y un grupo experimental. Las variables que se utilizan pueden ser dependientes (características que se quieren medir o el objeto de estudio del investigador) y las independientes (factor manipulado por el investigador). Además se debe controlar todas las demás variables que puedan influir en el estudio (variables extrañas).

Las variables dependientes consideradas en el estudio fueron: olor, color, concentración y duración. Las independientes: concentración y aditivo. Entre las consideradas como extrañas se tienen: asepsia del equipo, material y reactivo. ${ }^{2}$

\section{Resultados y discusión}

Los compuestos naturales: orégano (Origanum vulgares), clavo de olor (Syzygium aromaticum), comino (Cuminum cyminum), y ajo (Allium sativum) se adquirieron en el mercado local. Todos se sometieron a lavado y secado en seco y radiados con luz ultravioleta para eliminar cualquier agente patógeno adherido a la superficie del tejido vegetal. Todo el material se procesó seco, pesando cantidades de un kilogramo por especie vegetal seleccionada como persevante natural.

\section{Obtención de aceites esenciales}

Para la obtención de aceites esenciales se estableció el uso de dos técnicas; destilación simple y destilación por arrate de vapor. La destilación simple consistió en colocar en un balón de destilación de $2000 \mathrm{ml}$ el tejido vegetal pulverizado mezclado con agua. 
Este proceso fue aplicado a orégano (Origanum vulgares), clavo de olor (Syzygium aromaticum), comino (Cuminum cyminum), por su estructura, y su fácil pulverizado manual con el mortero y pistilo, pesando la cantidad de 1000 gramos por muestra de tejido vegetal.

La destilación simple permite que mediante la aplicación de calor directo a la mezcla de tejido vegetal y agua purificada, se calienten al mismo tiempo los compuestos con un punto de ebullición menor al del agua. Así, pasarán de la mezcla al estado gaseoso y se condensarán en el tubo, pasando al estado líquido. Al final se forma una mezcla oleosa donde el aceite esencial se separa por mezcla con otro aceite virgen, utilizando la miscibilidad y densidad mediante embudo de separación. Los rendimiento de aceite esencial mediante este método son de aproximadamente $2 \mathrm{ml}$ por cada 100 gramos de tejido vegetal.

Igualmente este proceso fue aplicado al orégano (Origanum vulgares), clavo de olor (Syzygium aromaticum) y comino (Cuminum cyminum). Para aumentar la superficie de contacto del tejido vegetal se pulverizó la muestra seca con ayuda del mortero y pistilo. La obtención de aceites se logra mediante la destilación de arrastre de vapor. Sin embargo, el rendimiento por cada 100 gramos de tejido vegetal no fue muy alentador: se obtuvo un rendimiento de 2.0 mililitros de aceite esencial por 100 gramos, en un periodo de destilación de cuatro horas.
En el proceso de destilación por arrastre de vapor hubo un problema, que resultó al separar el aceite del medio acuoso destilado. La parte acuosa estaba en mayor proporción, y el aceite inmiscible se adhirió a las paredes del embudo de separación. Para obtener aceite en mayor proporción y separarlo efectivamente, se efectuó la adición de un aceite virgen de canola o de oliva, para aumentar la capacidad separadora de la capa acuosa.

Debido a que el ajo es un bulbo con mucha humedad, no se pudo utilizar seco y tampoco se pudo calentar directamente debido a su fácil combustión. Esto hizo necesario que su extracción fuera diferente al método empleado con las otras especies vegetales utilizadas en la investigación. El mecanismo manejado fue a través del extractor Soxhlet.

Este proceso consistió en calentar agua hasta su punto de ebullición; los vapores ascendieron a través de la columna hasta el condensador; luego descendieron hasta el dedal de separación que contenía los bulbos de ajo (conocidos como dientes de ajo). Como estaba caliente, disolvió los aceites, y a medida que se llenaba el dedal de agua, iba ejerciendo disolución hasta que llegó al nivel del sifón; éste se abrió y llegó al balón de calentamiento, repitiéndose el proceso de manera cíclica. En esta separación solo se realizaron tres ciclos para evitar descomposición del ajo.

Para separar el aceite esencial del destilado acuoso, se procedió a utilizar aceite virgen de 
canola o de oliva; y a continuación se extrajo mediante el uso de un embudo de separación, el aceite esencial obtenido en el proceso. Es importante aclarar que del aceite virgen se debe conocer su pureza y densidad, para que al momento de separar la capa oleosa de la acuosa, se pueda determinar la concentración en la que se encuentran los aceites esenciales.

En cuanto al rendimiento del aceite de clavo de olor, este es mayor que los obtenidos con los demás componentes naturales involucrados en esta investigación. Obteniéndose 6 mililitros de aceite esencial puro por cada 100 gramos de clavo de olor pulverizado.

Tabla 1. Concentraciones y densidades

\begin{tabular}{|c|c|c|}
\hline Compuesto & $\begin{array}{c}\text { Densidad } \\
(\mathbf{g} / \mathbf{m l})\end{array}$ & $\begin{array}{c}\text { Concentra- } \\
\text { ción } \%(\mathbf{p} / \mathbf{v})\end{array}$ \\
\hline Aceite de orégano & 0.92 & 0.4 \\
\hline Aceite de ajo & 0.94 & 2.4 \\
\hline Aceite de clavo de olor & 0.97 & 5.4 \\
\hline Infusión de orégano & 1.01 & 1.24 \\
\hline Infusión de ajo & 1.02 & 2.24 \\
\hline Infusión de clavo de olor & 1.01 & 1.24 \\
\hline
\end{tabular}

No se determinó la concentración del aceite de comino debido a que su rendimiento fue menor, con un volumen de $0.3 \mathrm{ml}$ por cada 100 gramos de tejido vegetal.

Los análisis microbiológicos realizados durante esta investigación consistieron en pruebas de bacterias Coliformes, Coliformes Fecales, Escherichia Coli, recuento de bacterias Heterótrofas Mesófitas y Pseudomonas. Las técnicas empleadas fueron las de Tubos Múltiples (NMP),
Placas de Pretriflim y Placas Petri en recuento de colonias (UFC). (Ver tabla 2)

Se observó que los mejores inhibidores de crecimiento bacteriano son el clavo de olor y el orégano. Se efectuaron comparaciones simultáneas de los aditivos químicos y persevantes naturales, obteniéndose buenos resultados con los persevantes naturales, los que se muestran en las siguientes tablas. (Ver tabla 3)

Los resultados de la tabla 4 corresponden a compuestos extraídos de los tejidos vegetales, y a las pruebas realizadas para obtención de la formula requerida del persevante natural. Luego de estos resultados se decidió eliminar el ajo, el clavo de olor y el comino, y trabajar únicamente con el orégano en esencia.

\section{Esencia de orégano}

Para la obtención de esencia de orégano se procedió a utilizar hojas secas que no presentaban en la superficie crecimientos de hongos por efecto de humedad. Se pesó un kilogramo; se introdujo en una olla de presión de acero inoxidable de 5 litros previamente esterilizada; se agregaron 3 litros de agua purificada; colocándose al fuego a cocción por un periodo de 30 minutos; se dejó enfriar y se filtró en malla teflón con presión hasta dejar el sólido seco. El volumen promedio de esencia resultante es de unos 2300 mililitros, con una densidad de $1.23 \mathrm{~g} / \mathrm{ml}$ y una concentración del 24\% (p/v). Estos resultados pueden variar según el volumen de agua y la calidad del orégano. 
Tabla 2. Resultados microbiológicos de pruebas en preservantes naturales en conteos de 24 y 48 horas de incubación

\begin{tabular}{|c|c|c|c|c|c|}
\hline Producto & Coli tot & Coli. Fec. & E.coli & Heterotrofas & Pseudomonas \\
\hline Aceite de orégano & $<1.1$ & $<1.1$ & Negativo & 2UFC & Negativo \\
\hline Esencia de orégano & $<1.1$ & $<1.1$ & Negativo & $<1$ UFC & Negativo \\
\hline $\begin{array}{c}\text { Aceite de ajo } \\
\text { Aceite de clavo de } \\
\text { olor }\end{array}$ & $<1.1$ & $<1.1$ & Negativo & 6 UFC & Negativo \\
\hline $\begin{array}{c}\text { Esencia de clavo de } \\
\text { olor }\end{array}$ & $<1.1$ & $<1.1$ & Negativo & $<1$ UFC & Negativo \\
\hline Aceite de comino & $<1.1$ & $<1.1$ & Negativo & 12UFC & Negativo \\
\hline Esencia de comino & $<1.1$ & $<1.1$ & Negativo & 123UFC & Negativo \\
\hline
\end{tabular}

Tabla 3. Número y tipo de muestras analizadas

\begin{tabular}{|c|c|}
\hline Procesado cárnico & Número de muestras \\
\hline Chorizo Argentino & 8 \\
\hline Jamón Virginia & 12 \\
\hline Jamonada & 9 \\
\hline Salame Imperial & 9 \\
\hline Salchicha & 9 \\
\hline
\end{tabular}

Tabla 4. Resultados de pruebas microbiológicas y comparación con preservantes químicos

\begin{tabular}{|c|c|c|c|c|}
\hline \multirow{2}{*}{ Tipo } & \multirow{2}{*}{ Preservantes utilizados } & \multicolumn{3}{|c|}{ Parámetros microbiológicos } \\
\cline { 3 - 5 } & & Aerobios & Coliformes & E. Coli \\
\hline \multirow{3}{*}{ Infusión } & Orégano & 1520 & 1 & 0 \\
\cline { 2 - 5 } & Químico & 2390 & 1 & 0 \\
\cline { 2 - 5 } & Aceite Esencial & 45200 & 59 & 8 \\
\hline \multirow{2}{*}{ Químico } & Sal de Cura & 137950 & 107 & 0 \\
\hline \multirow{3}{*}{ Aceite Esencial } & Orégano & 1520 & 1 & 0 \\
\cline { 2 - 5 } & Ajo & 2390 & 1 & 8 \\
\cline { 2 - 5 } & Clavo de olor & 145200 & 59 & 14 \\
\hline Químico & Sal de Cura & 162950 & 107 & \\
\hline
\end{tabular}




\section{Análisis experimental}

Se analizaron cuatro muestras de jamonada de diferentes concentraciones y códigos, quedando de la siguiente manera

Tabla 5. Muestras, persevantes y concentraciones empleadas

\begin{tabular}{|c|c|c|}
\hline Código de muestra & Preservante & Concentración \\
\hline 001 & Orégano & $0.032 \%$ \\
\hline 002 & Sal de Cura & $0.0013 \%$ \\
\hline 003 & Orégano & $0.022 \%$ \\
\hline 004 & Orégano & $0.027 \%$ \\
\hline
\end{tabular}

\section{Conclusiones}

Los análisis se realizaron evaluando las características organolépticas de los productos y su parecido al estándar. Los parámetros a evaluar fueron los siguientes: color, olor y textura.

Tabla 6. Características organolépticas con la nueva formulación

\begin{tabular}{|c|c|c|c|}
\hline Muestra & Color & Olor & Textura \\
\hline 001 & Beige claro & Característico & Característico \\
\hline 002 & Rosado claro & Característico & Característico \\
\hline 003 & $\begin{array}{c}\text { Beige claro y puntos de } \\
\text { restos de orégano }\end{array}$ & Característico & Característico \\
\hline 004 & Beige claro & Característico & Característico \\
\hline
\end{tabular}

En cuanto a las características de color y textura, no existe diferencia entre las muestras analizadas. En lo que respecta al color, se recomienda el uso de colorante para asemejarlo al color de la jamonada existente en el mercado, ya que es un color mucho más suave que el característico. (Ver tabla 7).

Los resultados permitieron establecer que la concentración de esencia de orégano a usar en productos cárnicos procesados puede ser a partir del $0.027 \%$, en correspondencia a los parámetros especificados por la normativa salvadoreña NSO 67.02.13:98 Carne y Productos Cárnicos Embutidos Crudos y Cocidos. Este hallazgo es importante, considerando que la esencia de orégano obtenida en el proceso experimental cumple las mismas expectativas preservantes que la sal de cura.

Para la incorporación del aporte nutricional adicional, se pesaron 250 gramos de semilla de chan (Salvia hispánica L.) limpia y seca; se introdujeron en un recipiente esterilizado de 
acero inoxidable de 5 litros con tapa; se agregaron $2500 \mathrm{ml}$ de agua purificada y ozonizada, de manera que cubriera todo el contenido de la semilla; se dejó reposar por un periodo de 6 horas aplicándole agitación cada hora por unos tres minutos.

Posteriormente, luego de transcurrido el tiempo suficiente para que la semilla de chan (Salvia hispánica L.) se hidratare, con ayuda de la espátula o cuchara de acero inoxidable, se introdujo a la manga de malla teflón; cerrándola y colocándola en el dispositivo de filtro prensa; se comprimió de manera que se separar la capa untuosa sobre la semilla de chan, y saliera a través de la malla un líquido espeso color gris, que se colectó en la canaleta de recolección. Este proceso se continuó hasta extraer todo el contenido de ácidos grasos. Los rendimientos son muy buenos, a razón de 1.5 litros por cada 250 gramos a una concentración de $24 \%$ en peso, y con una densidad de $1.24 \mathrm{~g} / \mathrm{ml}$. Este valor varía según el volumen de agua empleado. (Ver tabla 8 )

\section{Costos de producción de esencia de orégano y chan}

Para la producción de la esencia de orégano, se requieren los materiales mostrados en la tabla 9.

Los costos indirectos, relacionados con el consumo de combustible y mano de obra, se calcularon en \$5.17. Para producir 1.8 litros de esencia de orégano se gastaron $\$ 6.05$ (costos directos) más \$ 5.17 (costos indirectos). El valor total del producto es de $\$ 11.22$ a una concentración del $23 \%$ de compuestos activos disueltos. El costo por mililitro es de $\$ 0.006$, aproximadamente $\$ 0.01$. (Ver tabla 10).

Para producir 1.5 litros de extracto oleoso de chan se gastaron $\$ 9.05$ (costos directos) más $\$ 5.17$ (costos indirectos). El valor total del producto obtenido es de $\$ 14.22$ a una concentración del $24 \%$ de compuestos activos disueltos. El costo por mililitro es de $\$ 0.009$, aproximadamente $\$ 0.01$.

\section{Prueba para degustación y rechazo}

Para realizar esta prueba de los procesados cárnicos con la aplicación de los persevantes naturales se utilizaron las siguientes presentaciones de chorizo, jamón, jamonada y salami. Se tomó una muestra de doce personas, $40 \%$ del género femenino y $60 \%$ masculinos, estableciéndose los siguientes criterios para la degustación o rechazo de los productos elaborados: apariencia, olor, color, textura gustativa y sabor. (Ver tabla 11) 
Tabla 7. Análisis microbiológico de esencia de orégano en la nueva formulación de los procesados cárnicos

\begin{tabular}{|c|c|c|c|}
\hline Muestra & Aerobios mesofilos & Coliformes totales & Escherichia coli \\
\hline 001 & $120 \mathrm{UFC} / \mathrm{g}$ & $0 \mathrm{UFC} / \mathrm{g}$ & $0 \mathrm{UFC} / \mathrm{g}$ \\
\hline 002 & $100 \mathrm{UFC} / \mathrm{g}$ & $0 \mathrm{UFC} / \mathrm{g}$ & $0 \mathrm{UFC} / \mathrm{g}$ \\
\hline 003 & $13,000 \mathrm{UFC} / \mathrm{g}$ & $12 \mathrm{UFC} / \mathrm{g}$ & $2 \mathrm{UFC} / \mathrm{g}$ \\
\hline 004 & $116 \mathrm{UFC} / \mathrm{g}$ & $0 \mathrm{UFC} / \mathrm{g}$ & $0 \mathrm{UFC} / \mathrm{g}$ \\
\hline
\end{tabular}

Tabla 8. Análisis microbiológico de extracto de la semilla de chan

\begin{tabular}{|c|c|c|c|c|c|}
\hline Producto & Coli tot & Coli. Fec. & E.coli & Heterotrofas & Pseudomonas \\
\hline $\begin{array}{c}\text { Extracto de la } \\
\text { semilla de chan }\end{array}$ & $<1.1$ & $<1.1$ & Negativo & 34 UFC & Negativo \\
\hline
\end{tabular}

Tabla 9. Costos de esencia de orégano

\begin{tabular}{|c|c|c|c|}
\hline Descripción & Cantidad & Costo unitario & Total \\
\hline Hojas fresca de orégano & Un Kilo & $\$ 5.00$ & $\$ 5.00$ \\
\hline Agua ozonizada & Tres Litros & $\$ 0.35$ & $\$ 1.05$ \\
\hline \multicolumn{2}{|r}{} & Total & $\$ \mathbf{6 . 0 5}$ \\
\hline
\end{tabular}

Tabla 10. Costos de esencia de chan

\begin{tabular}{|c|c|c|c|}
\hline Descripción & Cantidad & Costo unitario & Total \\
\hline Semilla seca de chan & 0.25 kilogramos & $\$ 8.00$ & $\$ 8.00$ \\
\hline Agua ozonizada & Tres Litros & $\$ 0.35$ & $\$ 1.05$ \\
\hline \multicolumn{2}{|l}{} & Total & $\$ 9.05$ \\
\hline
\end{tabular}

Tabla 11. Resultados de los criterios de degustación o rechazo

\begin{tabular}{|c|c|c|c|c|c|c|c|c|c|}
\hline \multicolumn{10}{|c|}{ Criterios } \\
\hline \multicolumn{2}{|c|}{ Apariencia } & \multicolumn{2}{|c|}{ Olor } & \multicolumn{2}{|c|}{ Color } & \multicolumn{2}{|c|}{ Textura gustativa } & \multicolumn{2}{|c|}{ Sabor } \\
\hline 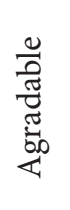 & 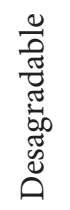 & $\begin{array}{l}\stackrel{0}{0} \\
\stackrel{0}{0}\end{array}$ & $\frac{0}{\pi^{\pi}}$ & $\underset{\stackrel{8}{ \pm}}{\stackrel{8}{ \pm}}$ & $\frac{0}{\sum^{\frac{\pi}{2}}}$ & 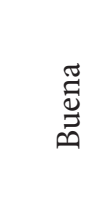 & 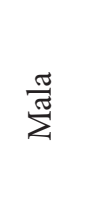 & $\underset{\stackrel{0}{0}}{\stackrel{0}{\overrightarrow{0}}}$ & $\frac{0}{\sum^{\frac{\pi}{3}}}$ \\
\hline 10 & 2 & 12 & 0 & 9 & 3 & 12 & 0 & 12 & 0 \\
\hline
\end{tabular}




\section{Referencias}

Aligiannis, N., Kalpoutzakis, E., Mitaku, S., y J, I. C. I. (2001). Composition and Antimicrobial activity of the essential oils of two Origanum species. Agric. Food Chem.

Andrade-Cuvi, M. (2008). Relación entre la capacidad antioxidante y el desarrollo del daño por frio en pimientos. Efecto de la radiación UV-C. Tesis. Facultad Ciencias Exactas. Universidad Nacional de la Plata.

Argentina, Administración Nacional de Medicamentos Alimentos y Tecnología Médica (s.f.). Guía de interpretación de resultados microbiológicos. Recuperado de http://www.anmat.gov.ar/alimentos/Guia_de_interpretacion_resultados_microbiologicos.pdf

Aracena, E. (2007). Evaluación del cambio de color en carne de canales bovinas mantenidas en cámara frigorífica con exposición a ozono gaseoso. (Médico Veterinario), Universidad de Concepción, Chillán- Chile.

Arango, C., y Restrepo, D. (2008). Microbiología de la carne. Recuperado de http://es.scribd.com/ doc/8717475/Cap-1-MicrobiologIa de-La-Carne

Arcila, C., G Loarca, Lecona, S., y González, E. (2004). El orégano: propiedades, composición y actividad biológica de sus componentes. 54, 100-110. Recuperado de http://www.scielo.org.ve/scielo. php?script=sci_arttext\&pid=S000406222004000100015\&lng=es\&nrm=iso

Ávila, A., Torres, J., Gastélum, M., y Nevárez, G. (2002). Antioxidant and Antimicrobial capacity of Mexican Orégano. IFT Annual Meeting.

Bejarano, A., y Silva, A. (2010). Estabilidad del helado de crema. Recuento de Coliformes totales en cajas Petri. (Tecnólogo en alimentos), ESPOL, Guayaquil-Ecuador. Recuperado de http://www. dspace.espol.edu.ec/71bitstream/123456789/9096/1/Estabilidad\%20del\%20helado\%20de\%20 crema\%20de\%20leche.pdf

Bell, B., Goldfolt, M., Griffin, P., Davis, M., Gordon, Tarr, Wells. (1994). A multistate outbreak of Escherichia coli O157:H7-associated bloody diarrhea and hemolytic uremic syndrome from hamburgers: the Washington experience. Recuperado de http://www.seimc.org/control/revisiones/ bacteriologic/o157.pdf 
Bello, J. (2000). Ciencia Bromatológica. Principios generales de los alimentos. Madrid. Díaz de Santos Bonilla, J.M. Presentación ppt, embutidos, UNICAES, 2013

Burt, S. (2004). Essential oils: their antibacterial properties and potential Application in foods-a review International Journal of Food Microbiology, pp 223- 253.

Casp, A., y April, J. (2003). Procesos de conservación de alimentos (Ediciones Mundi Prensa ed. Vol. Segunda Edición). Madrid, España.

Domínguez, L., y Ros, C. (2007). Manipulador de Alimentos. Segunda Edición. La importancia de la higiene en la elaboración y servicio de comidas. pp 72

Elgayyar, M., Draughon, F., Golden, D., y Mount, J. (2001). Antimicrobial activity of essential oils from plants against selected pathogenic and saprophytic microorganisms.

Informe trimestral proyecto de investigación procesados cárnicos UNICAES-UNIVO 2014 trabajo realizado por Noshino, S, Bonilla, J.P.

Organización de las Naciones Unidas para la alimentación y la agricultura (2012). Grupo de productos cárnicos. Productos cárnicos procesados crudos Recuperado de http://www.fao.org/ ag/ags/industrias-agroalimentarias/carne-y-leche/grupos-de-productos-carnicos/es/ 